\title{
Early bifurcation in rotating fluid flow with free surface studied by axisymmetric numerical simulations
}

\section{Santos, M. B. L.; Sørensen, Jens Nørkær}

Published in:

Physics of Fluids

Link to article, DOI:

10.1063/1.869079

Publication date:

1996

Document Version

Publisher's PDF, also known as Version of record

Link back to DTU Orbit

Citation (APA):

Santos, M. B. L., \& Sørensen, J. N. (1996). Early bifurcation in rotating fluid flow with free surface studied by axisymmetric numerical simulations. Physics of Fluids, 8(11), 3057-3062. https://doi.org/10.1063/1.869079

\section{General rights}

Copyright and moral rights for the publications made accessible in the public portal are retained by the authors and/or other copyright owners and it is a condition of accessing publications that users recognise and abide by the legal requirements associated with these rights.

- Users may download and print one copy of any publication from the public portal for the purpose of private study or research.

- You may not further distribute the material or use it for any profit-making activity or commercial gain

- You may freely distribute the URL identifying the publication in the public portal 


\title{
Early bifurcation in rotating fluid flow with free surface studied by axisymmetric numerical simulations
}

\author{
M. B. L. Santos ${ }^{\text {a) }}$ and J. N. Sørensen ${ }^{\text {b) }}$ \\ Department of Fluid Mechanics, Technical University of Denmark, DK-2800 Lyngby, Denmark
}

(Received 19 May 1995; accepted 29 July 1996)

\begin{abstract}
Simulations of a fluid rotating inside a partially open cylindrical cavity, performed by numerical solution of the unsteady axisymmetric Navier-Stokes equations, are presented. The configuration consists of a cylindrical vessel holding the fluid, which is entrained into motion by a rotating lid. This one is a coaxial disk in contact with the fluid surface but without covering it entirely. The study focuses on the occurrence of time-dependent flow, more specifically, the first transition to unsteadiness, by considering cavity cases with different amounts of free surface, for a fixed aspect ratio. By following the time evolution of a few arbitrarily chosen dynamical variables as a function of the Reynolds number, the location of this first Hopf bifurcation is obtained for a collection of cavity cases. Results show a rather strong influence of the free surface both on the onset of the unsteadiness and on the dynamical features of the flow. () 1996 American Institute of Physics. [S1070-6631(96)02411-7]
\end{abstract}

\section{INTRODUCTION}

The classical problem of a viscous fluid rotating inside a closed cylindrical cavity attracts a renewed interest in recent years. A common motivation among these experimental ${ }^{1,2}$ and numerical simulations $\mathrm{s}^{3,4}$ is the suitability of the driven cavity as a model system to explore the routes to chaos and turbulence.

A closely related system is a fluid rotating within a partially open cylindrical cavity of radius $R_{c}$. Figure 1 illustrates a particular configuration. Here the fluid, which fills the cavity up to the height $H$, is driven by a cover lid, or rotating "disk" of radius $R_{0}$, usually smaller than $R_{c}$ (that is, the case of the closed cavity appears as a particular one, for which $R_{0}=R_{c}$ ). The disk rotates at a rate $\Omega_{0}$ and barely dips the fluid surface, which is taken as flat.

From the viewpoint of applications, this is an important configuration, as it represents an idealized model for the Czochralski crystal puller. As the Czochralski method is the preferred one for growing silicon, obvious industrial interests have motivated a vast literature on this, so-called, bulk flow model (see, for example, the review article by Langlois ${ }^{5}$ ).

In a recent survey by one of us, ${ }^{6}$ some main aspects reflecting the state-of-art of this subject were emphasized, three which are worth mentioning here: (1) 2-D simulations $^{7-9}$ which detail the effects on the flow due to the crystal rotation, the crucible rotation, and the temperature; (2) experimental simulations, featuring the appearance of nonaxisymmetric flow, ${ }^{10,11}$ with some qualitative information about the transition to unsteady flow; (3) 3-D simulations, mostly regarding the appearance of symmetry breaking patterns. $^{12-14}$

Nevertheless, we remark that the approaches used in the Czochralski literature tend to be those of the CFD exclusively. In comparison with the literature on the related prob-

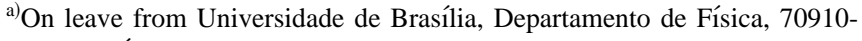
900 Brasília, DF, Brazil.

${ }^{b)}$ Author to whom correspondence should be addressed.
}

lem of the driven (closed) cavity, the use of concepts and techniques of the modern theory of dynamical systems and chaos in Czochralski flow studies remains scarce. As an example of such bifurcation theory approach, we quote a study of Bottaro and Zebib, ${ }^{14,15}$ about thermal convection.

The present paper, in contrast, deals with forced convection, due to disk rotation only. That is, the temperature is considered the same everywhere. In order to simulate the flow numerically, a previously developed ${ }^{16}$ high-order, finite-difference discretization code to solve the axisymmetric Navier-Stokes equations was employed. The computations were carried out on a Cray C92A machine at the UNI-C, DTU. In the case of the closed cavity, visualization experiments by Escudier ${ }^{1}$ have shown that even well into the unsteady domain the flow presents only negligible departure from axisymmetry. As mentioned above, the experimental knowledge on the partially open cavity is limited. It is known, however, that experiments on a totally open cavity, ${ }^{17}$ driven by the bottom, also confirm this feature. Therefore, the use of a 2-D code in this context appears to be fairly justifiable.

Specifically, the article aims to characterize the early transition to unsteadiness, which is generally toward an oscillatory, harmonic solution, as a function of the Reynolds number defined on the basis of the radius of the disk, i.e., $\operatorname{Re}=\Omega_{0} R_{0}^{2} / \nu$, with $\nu$ being the kinematic viscosity of the fluid. The computations were carried out for a fixed aspect ratio $\left(\lambda=H / R_{c}=2\right)$, by considering different values of the radius ratio, defined to be the ratio $\zeta$ between the disk radius and the cylinder radius, that is, $\zeta=R_{0} / R_{c}$. This allows to study the effect of the free surface on the critical Reynolds number associated with the bifurcation.

Only a few mentions are made in the paper to further unsteady solutions (double harmonics, etc.). It is expected that this study contributes as a first step for future investigations aiming to characterize the routes to chaos in these Czochralski-like flow systems. 


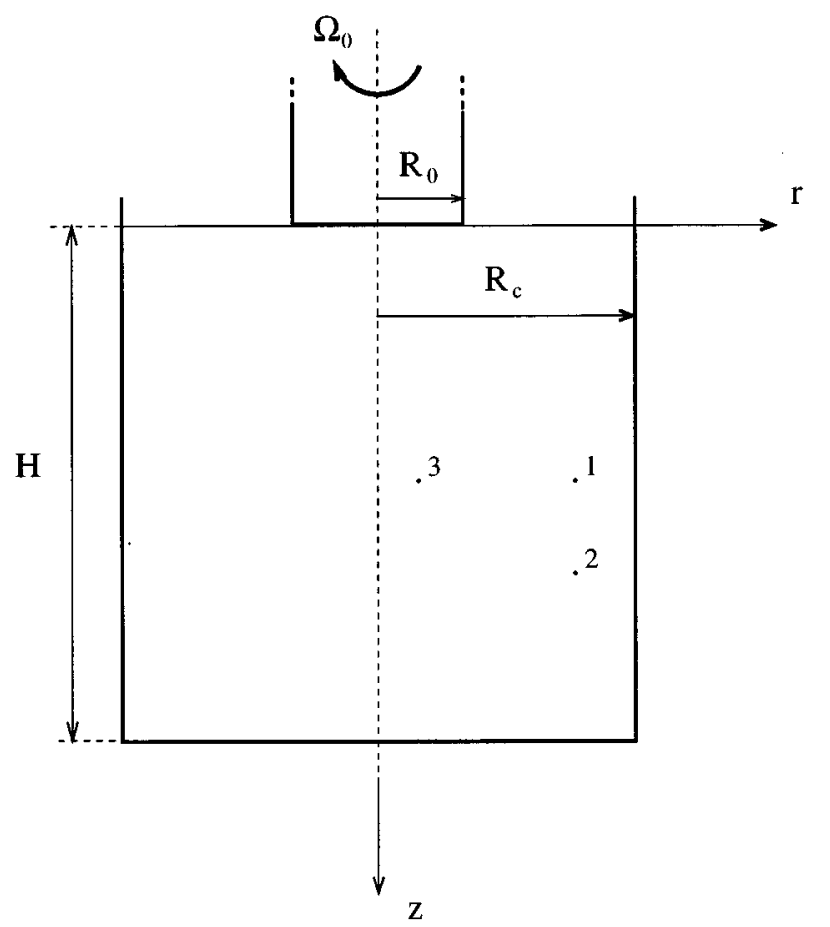

FIG. 1. Side-view sketch of an idealized Czochralski growth device: The rotating crystal (or "disk," of radius $R_{0}$ ) only dips the surface of the liquid, which is held by the cylindrical container (radius $R_{c}$ ); 1,2,3: locations of the monitor points used for probing the time series.

\section{PHYSICAL CONSIDERATIONS}

\section{A. Flow mechanism}

In all situations considered in this paper, the rotating disk is the only force driving the flow. Although the role played by the free surface should not be underestimated, the basic flow mechanism remains essentially like that for the closed cavity situation, and may be summarized as follows (see Sørensen and Christensen ${ }^{4}$ for a more detailed description). The viscous liquid adheres to the rotating disk (no-slip condition) producing a tangential fluid motion. This primary flow is subjected to centrifugal forces, which cause an upward secondary flow. The path is continued adjacently to the cylinder walls down to the bottom, where the radial pressure gradient (that usually tends to balance the centrifugal force) makes the flow converge toward the central axis. Briefly, the rotating lid acts like a fan sucking the stream up.

One important aspect to emphasize here is the boundary layer structure of the flow. In a simplified view the thickness $\delta$ of the layer of fluid "carried" by the disk can be estimated by ${ }^{18,19}$

$$
\delta \sim \sqrt{\frac{\nu}{\Omega_{0}}},
$$

and the radial and tangential velocity components have significant values only in a thin layer of thickness of order of $\delta$. The physical origin of the secondary flow is the centrifugal forces acting on that portion of the fluid confined in the rotating boundary layer (the Ekman layer). Although the above description is, strictly speaking, applicable to an infi-
TABLE I. Features of the meshes used in the computations.

\begin{tabular}{ccc}
\hline $\begin{array}{c}\text { Mesh } \\
\text { designation }\end{array}$ & $\begin{array}{c}\text { Number of nodes } \\
\left(N_{r} \times N_{z}\right)\end{array}$ & Density \\
\hline LD & $100 \times 200$ & 1 \\
HD & $200 \times 400$ & 2 \\
SD & $250 \times 500$ & 2.5 \\
\hline \hline
\end{tabular}

nite disk only, one may use the same results for a finite disk, provided that its radius is large compared with the thickness $(\sim \delta)$ of the boundary layer.

\section{B. Early bifurcation}

In a computational study on the driven (closed) cavity problem, Daube and Sørensen ${ }^{20}$ show that the transition from steady to periodic flow is consistent with the assumption of a supercritical Hopf bifurcation. Then, the amplitude modulus $\left|A_{f}\right|$ of the fluctuating part of a function $f(t)$ (or orbital radius, in a phase space diagram of two functions, say, $f$ and $g$, the time taken as a parameter) of any relevant flow variable, could be approximated ${ }^{18}$ by

$$
\left|A_{f}\right| \propto \sqrt{\mathrm{Re}-\mathrm{Re}_{\mathrm{cr}}}+O\left(\mathrm{Re}-\mathrm{Re}_{\mathrm{cr}}\right),
$$

which gives, sufficiently close to $\operatorname{Re}_{\mathrm{cr}}$,

$$
\left|A_{f}\right|^{2} \propto\left(\operatorname{Re}-\mathrm{Re}_{\mathrm{cr}}\right) \text {. }
$$

This linear relationship means that it is sufficient to compute $\left|A_{f}\right|^{2}$ for at least three values of Re slightly higher than the critical one. If the points are aligned, the extrapolation to zero of the corresponding straight line will give an estimate of the critical Reynolds number. In Sec. IV we shall use the same method to investigate the early transition for different cavity cases defined by the radius ratio $\zeta$.

\section{VALIDATION OF THE NUMERICAL CODE}

The flow is studied numerically by solving the axisymmetric Navier-Stokes equations in terms of stream functionswirl-vorticity variables $(\psi, \Gamma, \omega)$. The employed code was developed at LIMSI/CNRS in France (see, e.g., Daube et al. ${ }^{21}$ or Sbrensen and Ta $\mathrm{Phuoc}^{16}$ ) and has been tested against experimental observations in different situations (always for closed cavity), which we mention briefly, for reference. Steady-state stream function isolines have been compared with visualization experiments of Escudier. ${ }^{1}$ For example, at $\operatorname{Re}=1854$ and $\lambda=2$, it was found ${ }^{16}$ that a discretization of $91 \times 71$ was acceptably accurate. In addition, calculated velocity profiles have shown to be in good agreement with LDA measurements of Michelsen. ${ }^{22}$ Further validations of the code in the unsteady regime were made by Sørensen and Christensen ${ }^{4}$ by direct comparison of calculated structures due to particle injection and visualizations of Sørensen. ${ }^{23}$ Agreement is good at least up to $\operatorname{Re}=3000$, but comparisons become difficult for higher Reynolds number.

In the present study, tests were performed with different meshes, all having uniform spacing with $N_{r} \times N_{z}$ node points covering the calculation domain. Table I displays their characteristics. Most of the data have been obtained using a 100 $\times 200$ nodes mesh. Let us assign to this mesh a unity density, 


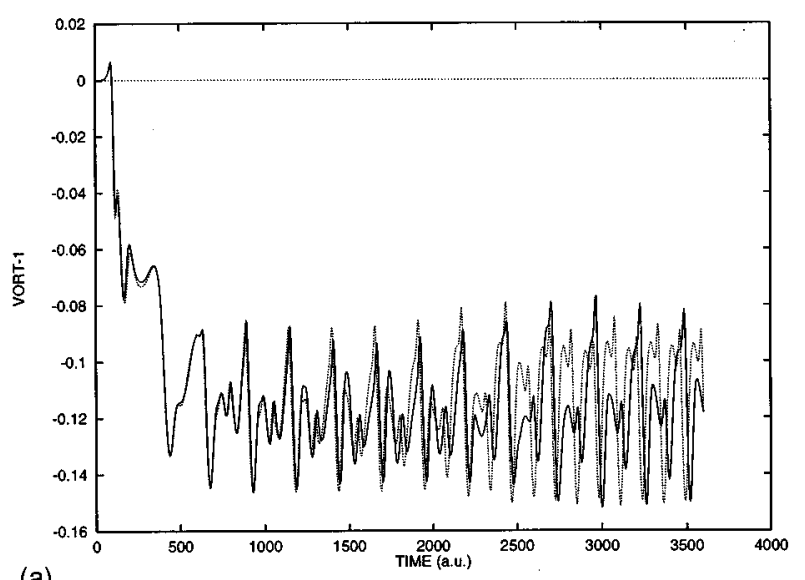

(a)

FIG. 2. Effect of mesh refining on the time series for the cavity case $\zeta=0.5, \operatorname{Re}=2000$ : (heavy lines) HD mesh (200 $\times 400$ node points); (dotted lines) LD mesh $(100 \times 200)$ : Comparisons between the results using both meshes for (a) the transient (start-up) regime and (b) the periodic regime.

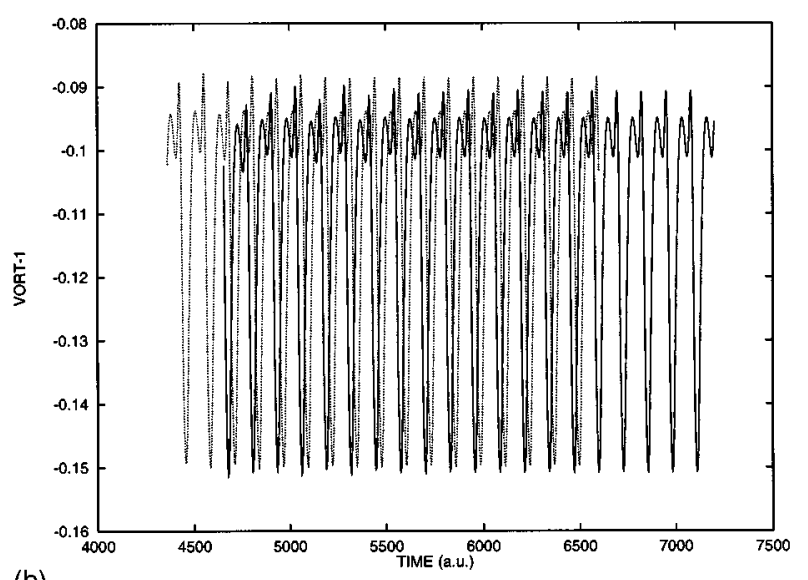

(b) $d=1$. Test computations (and part of the final results) have been performed on more dense meshes: $200 \times 400,250 \times 500$; or, in terms of node densities, $d=2$ and 2.5. Hereafter, the meshes used shall be referred by the acronyms LD, HD, and SD (which stand for "'low," "high,', and 'super', densities, respectively).

The CPU time necessary to obtain a stable solution varies according to the regime, type of solution and the proximity of the critical point. For the LD mesh, typical figures in the machine used (Cray C92A) are $5 \mathrm{~min}$ for a stationary solution and between $\frac{1}{2}$ to $1 \mathrm{~h}$ for an oscillatory solution. The effect of mesh refining on such figures is twofold. First, there is an increase of the number of nodes (that goes as $d^{2}$ ) and second, more iterations have to be done to compensate a decrease of the time step.

The figures given on the CPU time consumption are for (quasi) sinusoidal or two-harmonic oscillations. Solutions with three (or more) spectral components can be much longer to characterize ${ }^{4}$ and, of course, they were not systematically searched here. Some saving of computational time $(20 \%-30 \%)$ can be achieved by taking a previously obtained solution as the initial one, provided that the Reynolds numbers of the old and the new solutions are not very far $( \pm 100)$. Finally, the CPU time consumption increases dramatically as the transition is too closely approached.

Figure 2(a) and (b), which depicts the time-history of the vorticity on an arbitrary point in the flow domain, illustrate the fact that the periodic regime tends to be less requiring on mesh refining, as compared with the transient regime. Indeed, if one looks only into Fig. 2(a), the mesh HD seems to be necessary. However, as the stability is reached [Fig. 2(b)], it becomes clear that the mesh LD suffices for characterizing the steady-state oscillations of the $\zeta=0.5$ cavity case.

To conclude on the code validation, we have been brought, after an ensemble of tests performed with the different meshes of Table I, to the following operational conclusion:

(1) for $\zeta \geqslant 0.5$, use $\mathrm{LD}$;

(2) for $0.2 \geqslant \zeta \leqslant 0.3$, use HD.

\section{RESULTS AND DISCUSSION}

\section{A. Stationary regime}

Extensive simulations of the stationary regime of Czochralski flow are available in the literature, two of which have been quoted in the Introduction. Thus here we have chosen to show only few examples of flow visualizations, with a twofold purpose. First, to illustrate some basic features of the flow. And second, to give evidences of a vortex breakdown effect that, as far as we know, has not yet been discussed in connection with Czochralski numerical simulations.

Figure 3 shows streamline visualizations of the stationary flow for three examples of cavity cases $\zeta$ and Reynolds numbers. Note that only the right-hand side of the cavity meridional plane is shown, and also that the disk profile is sketched inside the computational domain, for pictorial purposes only. This domain was discretized using a $100 \times 200$ uniform mesh. The contours represent streamlines $\psi=$ const., which are tangent everywhere to the local velocity vector. In the case of Fig. 3(a), for $\zeta=0.3$ and $\operatorname{Re}=1300$, the pattern is

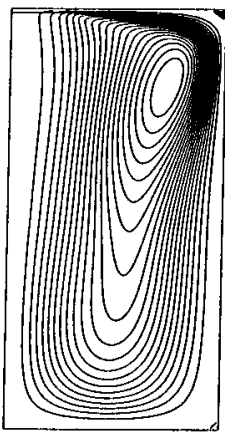

(a)

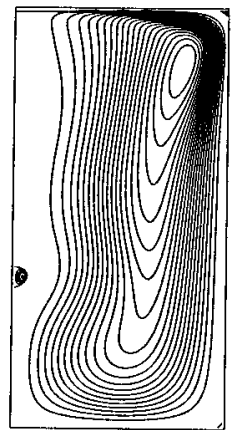

(b)

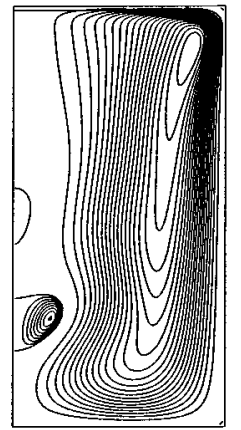

(c)
FIG. 3. Streamlines of the stationary flow in three cavity cases: (a) $\zeta \equiv R_{0} / R_{c}=0.3$, for $\operatorname{Re}=1300$; (b) $\zeta=0.5, \operatorname{Re}=1200$; (c) $\zeta=0.7, \operatorname{Re}=1500$. Figures show the right half-view of the meridional plane. The representation of the disk inside the computation domain is merely pictorial. In all cases, the sense of the main vortex is clockwise. 


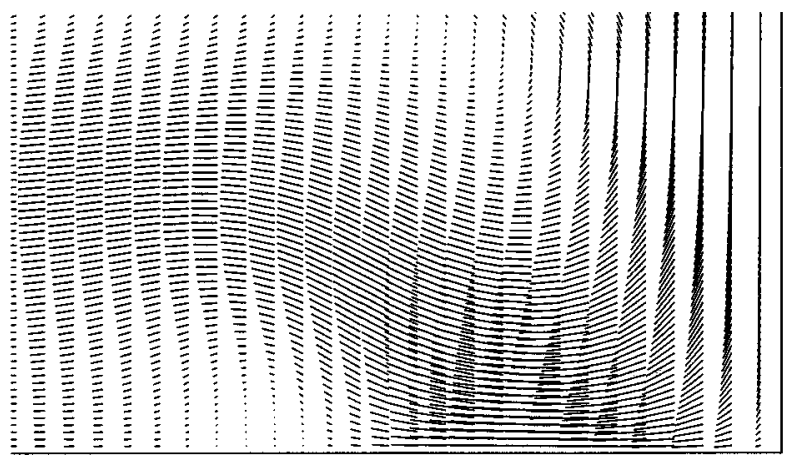

FIG. 4. Local velocity field visualization for $\zeta=0.5, \mathrm{Re}=1200$, featuring the small "bubble", seen in Fig. 3(b), which corresponds here to a rather clear area close to the axis (left side). The figure magnifies one small area of the meridional plane, in the corner defined by the axis and the cavity bottom.

monocellular, and corresponds to the qualitative features expected from the discussion of Sec. II. The vortex center is situated in the upper outer part of the meridional plane. Three regions can be roughly distinguished in the computation domain, one intermediate, limited by an outer region near the cylinder wall, and an inner region close to the cylinder axis. These last two exhibit a pronounced Taylor column effect, ${ }^{24}$ as usual for such moderately high Reynolds numbers, aside the end regions near the disk and the cavity bottom.

Now looking at Fig. 3(b), which shows the isolines for a cavity with less free surface, namely $\zeta=0.5$, some differences are worth noting. For instance, the inner streamlines appear undulated. In this example, $R e=1200$, a value not very far from that of the previous example, of Fig. 3(a). Actually, a comparison with the very same Reynolds value of that case is difficult to reach here by the approach of the bifurcation (see later), which makes the steady state very hard to reach.

The more striking feature of Fig. 3(b), however, is the recirculation bubble appearing around the cylinder axis, probably a reminiscence of the (much stronger) vortex breakdown effect known from the closed driven cavity. ${ }^{1,3,25}$ See also Fig. 4, that shows the local velocity field in the bubble region. Indeed, such core structures become larger (and eventually doubled) for higher $\zeta$ values [see Fig. 3(c), for $\zeta=0.7]$, whereas they are absent for open cavities with crystal radius $R_{0} \leqslant 0.3 R_{c}$. For such cases the flow pattern is rather like that of Fig. 3(a).

To conclude about stationary flow, the main features described here agree qualitatively well with previous Czochralski (isothermal) simulations available in the literature, except for the mentioned absence of vortex breakdown occurrences in those reports. For other aspect ratios see, e.g., the visualizations by Crochet $^{8}$ and Langlois. ${ }^{9}$

\section{B. Critical Reynolds number and characteristic times}

As suggested in Sec. II, the early transition is, in general, toward an oscillatory, harmonic solution. However, direct transition through more complicated states has been reported in a related rotor-stator system. ${ }^{26}$ In our computations there is no such occurrence, but it is noticeable that, for small
$R_{0} / R_{c}$, once the unsteadiness is reached, one arrives soon, by slightly increasing $\mathrm{Re}$, to quasiperiodic solutions. This should not be confused, however, with "tendency to instability,' ' in view that, for $\zeta<0.5$, it will appear from the data below that $\operatorname{Re}_{\mathrm{cr}} \rightarrow \infty$ for $\zeta \rightarrow 0$.

Specifically, within the scope of the present computations, what can be said is that the range of Reynolds numbers for sinusoidal solutions becomes progressively narrower, as $\zeta \rightarrow 0$. For instance, in the case $\zeta=0.2$, the contribution of higher frequency harmonics to the solution becomes quite important in an interval less than $\Delta \mathrm{Re}=50$, above $\operatorname{Re}_{\text {cr }}$, while, in all other cases studied, such important deviations of the sinusoidal behavior do not appear before $\left(\operatorname{Re}_{\mathrm{cr}}+300\right)$.

Following Sec. II, the quantitative characterization of the transition is made by "measuring" the peak-to-peak amplitude on stable time series (or, equivalently, the diameter of phase space portraits giving stable cyclic orbits) into the unsteady domain. Then, time series have been generated for the following sample variables:

$$
\omega_{1}, \omega_{2}, \omega_{3}, \psi_{1} \text {, and } \psi_{2} \text {. }
$$

Here, the labels ' 1 ', ' '2', and ' 3 ", refer to the three arbitrarily chosen monitor points, their coordinates $(r, z)$ in the meridional plane being (Fig. 1)

$$
\begin{aligned}
& \text { " } 1 \text { '” }=\left(R_{x}, z_{1}\right), " \text { ' } 2 \text { ', }=\left(R_{x}, z_{2}\right) \text {, and } \\
& \text { ' } 3 \text { '’ }=\left(\frac{1}{2} R_{0}, z_{1}\right),
\end{aligned}
$$

where

$$
R_{x}=\frac{3}{4} R_{c}, \quad z_{1}=\frac{1}{2} H, \text { and } z_{2}=\frac{2}{3} H .
$$

Note that the abscisse of point " 3 "' is the only one that does not scale with the cavity size, staying, for all cases, under the disk at half of its radius apart from the central axis. This particularity will reflect on the data issued for this point.

Hence, in terms of attractors and their topological nature, we are interested in determining the critical value of the dynamical parameter of our system $(\mathrm{Re})$ for which the attractor changes from a stable fixed point to a limit cycle, ${ }^{27}$ that is, the first bifurcation.

In spite of the differences detected by the effect of mesh refinement, no serious disagreement has been found in the critical Reynolds numbers evaluated after both the LD and HD computations in the cavity case $\zeta=0.3$. Such estimates were extracted from plots like those shown in Fig. 5(a) and (b), according to the method discussed in Sec. II (NB: in these plots, $\omega_{1}, \omega_{2}, \omega_{3}$, appear noted $v_{1}, v_{2}, v_{3}$, and $\psi_{1}, \psi_{2}$, are noted $p_{1}, p_{2}$, respectively).

The plots for these five variables behave in a rather different way. In particular, the curve representing the only time series probed at point 3 of the cavity $\left(\omega_{3}\right)$ has much stronger amplitudes, reflecting the turmoil in those regions just under the rotating disk (see Fig. 1). In spite of this diversity, however, all curves have the tendency of crossing the abscisses axis at some common point, according to the theoretical predictions. Of course, as the computational time grows exceedingly as the critical point is approached, the determination of $\mathrm{Re}_{\mathrm{cr}}$ always has to be done within some limited accuracy. 


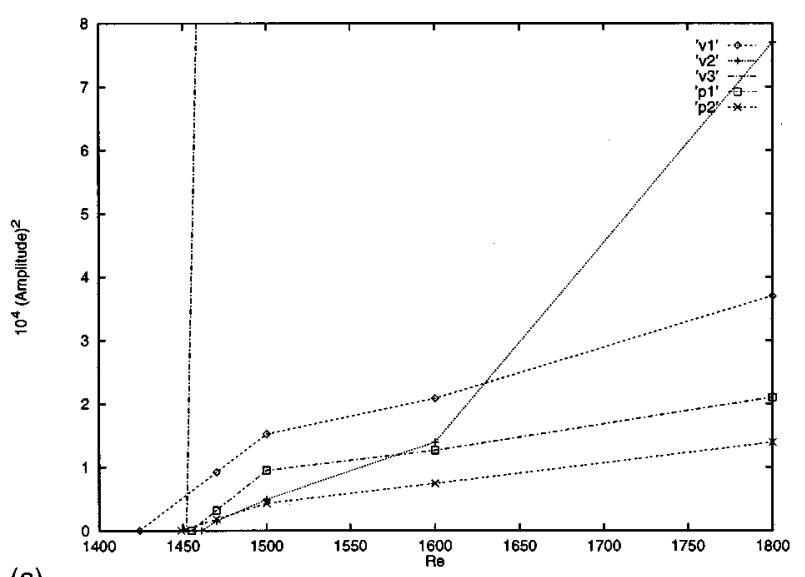

(a)

FIG. 5. $\mathrm{Re}_{\mathrm{cr}}$ estimates from squared (peak-to-peak) amplitude extrapolations on the time series. Comparison of the results with the (a) LD and (b) HD meshes for the cavity case $\zeta=0.3$. Conventions: See text.

Still, considering the case $\zeta=0.3$, after averaging the results of the five curves for the extrapolations prescribed by Eq. (2), we obtained $\mathrm{Re}_{\mathrm{cr}}=1448 \pm 7$ and $1380 \pm 26$ for the LD and the HD meshes, respectively. That less dispersion is present in the LD case, merely reflects a closer approach to $\mathrm{Re}_{\mathrm{cr}}$ adopted in the computations with this mesh. In both $\mathrm{Re}_{\mathrm{cr}}$ determinations the dispersion is less than $\pm 2 \%$. Nevertheless, the difference in the $\mathrm{Re}_{\mathrm{cr}}$ value itself is significant, and so we have chosen the HD value for the case $\zeta=0.3$ in the plot of $\operatorname{Re}_{\text {cr }}$ vs $\zeta$, shown in Fig. 6 .

This plot demonstrates that the free surface strongly affects the onset of the instability. For instance, the critical value for the closed cavity $(\zeta=1)$ almost doubles the value corresponding to the minimum $\mathrm{Re}_{\mathrm{cr}}$. This minimum value occurs for a disk radius that is half the length of the cylinder radius. Then $\mathrm{Re}_{\mathrm{cr}}$ raises again, as $\zeta$ decreases. This effect is accompanied by an increasingly slow behavior of the flow dynamics, as shown in Fig. 7, where the frequency of the oscillations just above $\operatorname{Re}_{\mathrm{cr}}$ is plotted against $\zeta$. It can be seen that the frequency decreases dramatically as $\zeta$ decreases, actually suggesting an ultimate limit to zero frequency.

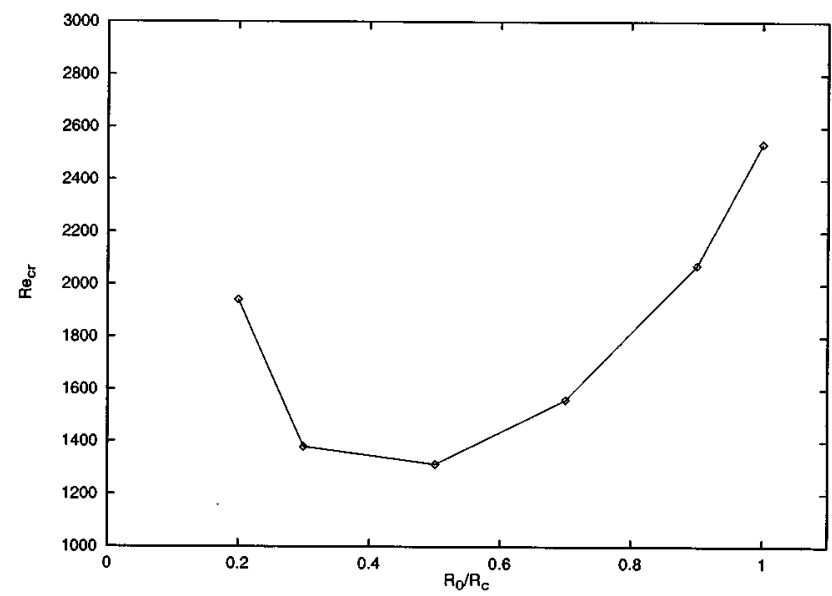

FIG. 6. $\operatorname{Re}_{\mathrm{cr}}$ versus the ratio of radii, $\zeta \equiv R_{0} / R_{c}$ : effect of the free surface on the critical Reynolds number for the early bifurcation to unsteady flow.

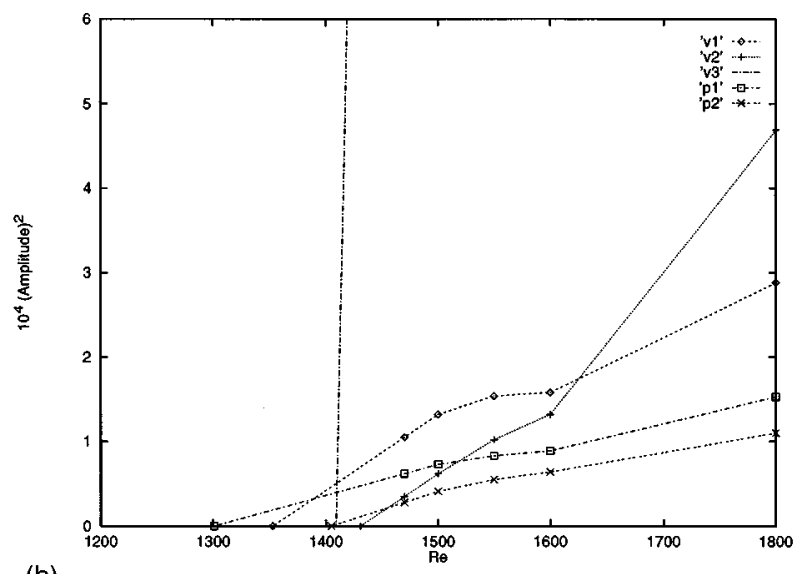

(b)

As an example of a simple physical argument supporting this idea, one could realize that if the container is allowed to grow (at a constant aspect ratio) the "information" (emitted by the exciting device, i.e., the disk) will require a longer time to propagate throughout the fluid.

However, the slowing down of the growing cavity is certainly stronger than this obvious linear effect, by the following reason. As the rotating disk is the only generator of perturbations in the system, it follows that as $R_{c}$ becomes large, most of the energy associated to the perturbations is used to excite modes of large wavelengths, which become more and more important as the size of the "box" grows. In other words, many reflections on the cavity walls tend to be necessary to "reconstruct'" the flow pattern whenever $R_{c} \gg R_{0}$, which means that additional time is needed to accomplish the cavity response. Naturally, such a "Fourier superposition view' is expected to hold only for linear systems, which is not rigorously the case. Nevertheless, for small $\zeta$ the flow is rather inviscid, hence the linear approximation should apply to some extent.

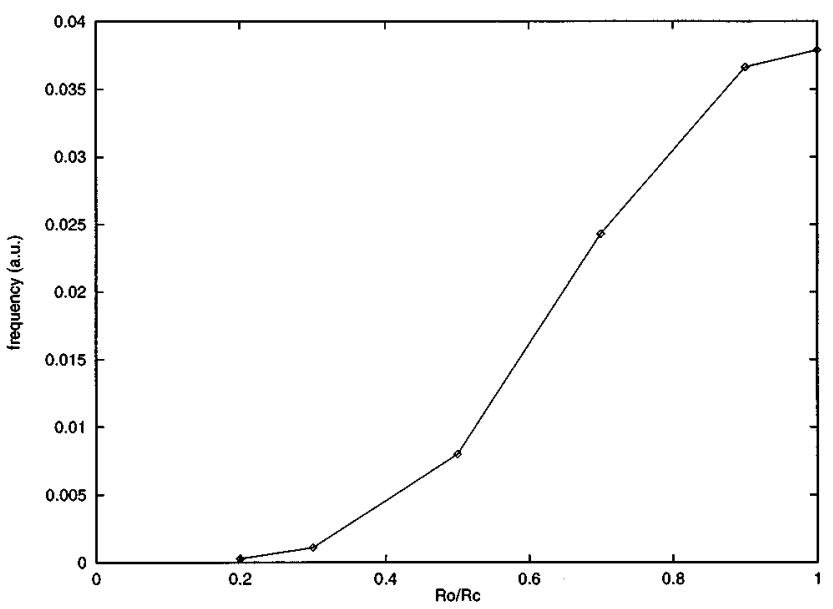

FIG. 7. Frequency of oscillations just above $\operatorname{Re}_{\mathrm{cr}}$ as a function of $\zeta$. 


\section{CONCLUSION}

To conclude, we presented results of numerical simulations of a rotating fluid flow in a cylinder with a partially open lid, which resembles a Czochralski-like geometry. Data were generated by employing a well-tested two-dimensional code to solve the Navier-Stokes equations subjected to axially symmetric boundary conditions.

Next, a bifurcation-type analysis of the time series data was performed, aiming to characterize the early transition to unsteadiness, that is, from steady to oscillatory flow. The computations were carried out for a fixed aspect ratio $(\lambda=2)$, as function of the ratio $\zeta$ between the disk radius and the cavity radius, in order to study the effect of the free surface on the critical Reynolds number associated with the bifurcation. This central result of the paper, embodied by Fig. 6, may have practical implications in Czochralski growth processes, in spite of the extreme simplicity of the present model. It predicts a "region" of maximum instability for oscillations (that is, low $\mathrm{Re}_{\mathrm{cr}}$ values), when the growing crystal attains a radius comparable to the half of the radius of the crucible (melt container). On the other hand, in the preceding "seed" stage of the crystal, the $\mathrm{Re}_{\text {cr }}$ values are rather elevated, allowing, in principle, for high rotation rates.

Finally, data on the dynamical properties of the flow as function of the radius ratio $\zeta$ were presented. The frequencies associated to the computed variables seem to tend to a zero limit as $\zeta \rightarrow 0$, and a plausibility argument for this behavior was given.

A final comment is worth making, concerning future work. Although the power of three-dimensional simulations is tempting, we point out that the possibilities of (much cheaper) two-dimensional simulations to characterize bifurcations are still unexplored in this context. For example, even in the particular case of the early bifurcation, there remain interesting issues to examine as the effect of different aspect ratios, as well as the inclusion of temperature effects. On the other hand, a natural continuation of the study made here would be to investigate further transitions, since the whole range of Reynolds numbers of interest has not been fully covered. Again compared with the closed cavity case, for which the state-of-art of bifurcation studies is in a much more advanced stage, it is worth mentioning that for higher Reynolds numbers a cascade of other effects arises ${ }^{2,28}$ before the flow becomes ultimately chaotic.

\section{ACKNOWLEDGMENTS}

The computer simulations, performed on the Cray C92A installed at UNI-C, in Lyngby, were financed by the Danish Technical Research Council. MBLS thanks CNPqConselho Nacional de Desenvolvimento Científico e Tecnológico (brazilian agency) for an one-year post-doctoral stipend. MBLS also wishes to express his gratitude to Professor Scheel Larsen and the staff of the AFM-DTU for the kind hospitality during his stay in Denmark. Special thanks are due to E. A. Christensen for stimulating remarks concerning bifurcations and the driven cavity problem, and to K. S. Hansen, D. Sørensen, and J. H. Walther for valuable computational help.
${ }^{1}$ M. P. Escudier, "Observations of the flow produced in a cylindrical container by a rotating endwall," Exp. Fluids 2, 189 (1984).

${ }^{2}$ C. H. Westergaard, P. Buchhave, and J. N. Sørensen, "PIV measurements of turbulent and chaotic structures in a rotating flow using an optical correlator," in Proceedings of the Sixth International Symposium on Applications of Laser Techniques to Fluid Mechanics-Lisbon, 20-23 July 1992 (Springer-Verlag, Berlin, 1992).

${ }^{3}$ J. M. Lopez and A. D. Perry, "Axisymmetric vortex breakdown, part 3. Onset of periodic flow and chaotic advection,' J. Fluid Mech. 234, 449 (1992).

${ }^{4}$ J. N. Sørensen and E. A. Christensen, "Direct numerical simulation of rotating fluid flow in a closed cylinder," Phys. Fluids 7, 764 (1995).

${ }^{5}$ W. E. Langlois, "Buoyancy-driven flows in crystal-growth methods," Annu. Rev. Fluid Mech. 17, 191 (1985).

${ }^{6}$ M. B. Lacerda Santos, "Czochralski bulk flow problem: physical aspects and a survey on the CFD literature," report AFM-94-05, Dept. of Fluid Mech., Techn. Univ. of Denmark, Lyngby, 1994.

${ }^{7}$ N. Kobayashi, "Hydrodynamics in Czochralski growth-computer analysis and experiments," J. Cryst. Growth 52, 425 (1981).

${ }^{8}$ M. J. Crochet, P. J. Woulters, F. T. Geyling, and A. S. Jordan, "Finiteelement simulations of Czochralski bulk flow," J. Cryst. Growth 65, 153 (1983).

${ }^{9}$ W. E. Langlois, "Digital simulation of Czochralski bulk flow in a parameter range appropriate for liquid semiconductors,' J. Cryst. Growth 42, 386 (1977).

${ }^{10}$ K. Kakimoto, M. Eguchi, H. Watanabe, and T. Hibiya, "Natural and forced convection of molten silicon during Czochralski single crystal growth," J. Cryst. Growth 94, 412 (1989).

${ }^{11}$ A. D. W. Jones, "Flow in a model Czochralski oxide melt," J. Cryst. Growth 94, 421 (1989).

${ }^{12}$ M. Mihelčić, K. Wingerath, and Chr. Pirron, “Three-dimensional simulations of the Czochralski bulk flow,' J. Cryst. Growth 69, 473 (1984).

${ }^{13}$ H.-J. Leister and M. Perić, "Numerical simulation of a 3D Czochralski melt flow by a finite volume multigrid-algorithm," J. Cryst. Growth $\mathbf{1 2 3}$, 567 (1992).

${ }^{14} \mathrm{~A}$. Bottaro and A. Zebib, "Three-dimensional thermal convection in Czochralski melt," J. Cryst. Growth 97, 50 (1989).

${ }^{15}$ A. Bottaro and A. Zebib, "Bifurcation in axisymmetric Czochralski natural convection," Phys. Fluids 31, 495 (1988).

${ }^{16}$ J. N. Sørensen, and Ta Phuoc Loc, "High-order axisymmetric NavierStokes code: Description and evaluation of boundary conditions," Int. J. Num. Meth. Fluids 9, 1517 (1989).

${ }^{17}$ A. Spohn, "Ecoulement et éclatement tourbillonnaires engendrés par un disque tournant dans une enceinte cylindrique," Thèse de Docteur de l'Université J. Fourier, Grenoble-I.

${ }^{18}$ L. D. Landau and E. M. Lifshitz, Fluid Mechanics, 2nd ed. (Pergamon, Oxford, 1987).

${ }^{19} \mathrm{H}$. Schlichting, Boundary Layer Theory, 7th ed. (McGraw-Hill, New York, 1979).

${ }^{20}$ O. Daube and J. N. Sørensen, "Simulation numérique de l'ecoulement périodique axisymétrique dans une cavité cylindrique," C. R. Acad. Sci. Paris 308-II, 463 (1989).

${ }^{21}$ O. Daube, Ta Phuoc Loc, P. Monnet, and M. Coutanceau, "Ecoulement instationnaire décollé d'un fluide incompressible autour d'un profil: une comparaison théorie-expérience,"' AGARD CP 386 (1985).

${ }^{22} \mathrm{~J}$. A. Michelsen, "Modeling of laminar incompressible rotating fluid flow," Ph.D. thesis, Technical University of Denmark, Lyngby, 1986.

${ }^{23}$ J. N. Sørensen, "Visualizations of rotating fluid flow in a closed cylinder,' report AFM-92-06, Dept. of Fluid Mech., Techn. Univ. of Denmark, Lyngby (1992).

${ }^{24}$ D. J. Tritton, Physical Fluid Dynamics, 2nd ed. (Oxford U.P., New York), Chap. 16.

${ }^{25}$ P. Buchhave, M. L. Jakobsen, C. H. Westergaard, and J. N. Sørensen, "PIV: measurement of the early transition of a rotating flow in a closed cylinder,' Laser Anemometry ASME J. 1, 45 (1991).

${ }^{26}$ O. Daube, N. Cousin, P. Le Quéré, and J. Pécheux, "Sur l'influence des effets de courbure sur la transition à l'instationnarité des écoulements interdisques," report 94-19, LIMSI-CNRS, Orsay, France (1994).

${ }^{27}$ J.-P. Eckmann, "Roads to turbulence in dissipative dynamical systems," Rev. Mod. Phys. 53, 643 (1981).

${ }^{28} \mathrm{E}$. A. Christensen, "Laminar-turbulent transition in the rotating driven cavity problem,' Ph.D. thesis, Technical University of Denmark, Lyngby, 1994. 